\title{
COLONIZAÇÃO DO “LITTER” DE Eleocharis sellowiana KUNTH. (CYPERACEAE) POR LARVAS DE CHIRONOMIDAE (DIPTERA) EM UM BREJO NO LITORAL DO ESTADO DO RIO DE JANEIRO
}

\author{
Jorge Luiz Nessimian \& Ana Lucia Henriques-de-Oliveira
}

\begin{abstract}
Eleocharis sellowiana Kunth litter colonization by Chironomidae (Diptera) larvae in a Rio de Janeiro state, Brazil, coastal marsh- The colonization process by macroinvertebrates of litter of Eleocharis sellowiana (Cyperaceae), the dominant macrophyte in the Itaipuaçú marsh, Maricá, Rio de Janeiro State, was studied. An experiment was carried out in the period from April to June, 1996. Twenty five samples of same weight (50 g) of leaves were deposited and 5 series of 5 samples were collected in a period of 70 days. Insects represented $51 \%$ of the macroinvertebrates and among them Chironomidae (Diptera) was the most important taxon with $58 \%$ (2347 individuals). Among Chironomidae, Chironomini (Chironominae) constituted the most abundant group with $63.1 \%$, followed by Tanypodinae with $34.3 \%$. Tanytarsini (Chironominae) had reduced participation (2.6\%). The genera encoutered were Chironomus, Polypedilum, Beardius (Chironomini), Tanytarsus (Tanytarsini), Clinotanypus, Ablabesmyia, Labrundinia, and Larsia (?) (Tanypodinae). Chironomus was the most important group with more than $50 \%$ of the total of chironomids sampled, followed by Larsia (?) with approximately $25 \%$. Chironomini, especially Beardius and Chironomus, presented growing abundance up to 42 days of
\end{abstract}

Departamento de Zoologia, Instituto de Biologia, Universidade Federal do Rio de Janeiro, Caixa Postal 68044, CEP: 21944-970 Cidade Universitária, Rio de Janeiro, RJ, Brasil. Email: nessimia@acd.ufrj.br

Com auxílio do CNPq, Faperj, Capes

Entomol. Vect. 12 (2): 159-172, 2005 
exposition, having a small retreat at the end of the experiment. The same growth happened with Tanypodinae, reaching a stabilization after 42 days. Tanytarsini (Tanytarsus sp.) became only significant at the end of the experiment. The relationships between the occupation of chironomid larvae, the decomposition process of $E$. sellowiana, the concentration of periphytic algae, and the hydrologic regime of the waterbody are discussed.

Key words: Chironomidae, larvae, colonization, macrophytes, marshes

\section{Resumo}

Foram observados os processos de colonização do "litter" de Eleocharis sellowiana (Cyperaceae), macrófita dominante no Brejo-canal de Itaipuaçú, Maricá, RJ, pela fauna de macroinvertebrados aquáticos. Realizou-se um experimento no período de abril a junho de 1996, depositando-se no corpo d'água, 25 amostras de mesmo peso (50 g de peso úmido) de folhas. Foram realizadas 5 retiradas de 5 amostras em um período de 70 dias. Insetos representaram $51 \%$ do total de macroinvertebrados colonizadores sendo Chironomidae (Diptera) o principal táxon com 58\%, totalizando 2347 indivíduos. Dentre os Chironomidae, Chironomini (Chironominae) constituiu o grupo mais abundante com 63,1\%, seguido de Tanypodinae com 34,3\%. Tanytarsini (Chironominae) apresentou participação reduzida (2,6\%). Os gêneros encontrados foram Chironomus, Polypedilum, Beardius (Chironomini), Tanytarsus (Tanytarsini), Clinotanypus, Ablabesmyia, Labrundinia e Larsia (?) (Tanypodinae). Chironomus foi o grupo mais importante com mais de 50\% do total, seguido de Larsia (?) com aproximadamente $25 \%$. Os Chironomini, especialmente Beardius e Chironomus apresentaram abundância crescente até 42 dias de exposição, havendo uma pequena queda ao final do experimento. $\mathrm{O}$ mesmo crescimento ocorreu com os Tanypodinae, havendo uma estabilização após 42 dias. A participação de Tanytarsini (Tanytarsus sp.) só tornou-se significativa no final do experimento. As relações entre a ocupação por larvas de Chironomidae, o processo de decomposição de E. sellowiana, a concentração de algas perifíticas e o regime hidrológico do corpo d'água são discutidas.

Palavras-chave: Chironomidae, larvas, colonização, macrófitas, brejos 


\section{Introdução}

Brejos estão entre os ambientes aquáticos mais produtivos, com densa vegetação e abundante fauna de macroinvertebrados, havendo uma relação positiva entre a quantidade e a riqueza desses dois componentes (Nessimian, 1995a). Embora macrófitas aquáticas tenham pouca importância como alimento enquanto vivas (Bruquetas de Zozaya \& Neiff, 1991), toda a dinâmica da comunidade de macroinvertebrados desses corpos d'água depende direta ou indiretamente da diversidade e do ciclo de vida das macrófitas presentes (Voigts, 1976; Nessimian, 1995a). Sua principal utilização como fonte de alimento se dá no processo de decomposição, durante o qual, de acordo com Suren \& Lake (1989), ocorrem maiores concentrações de nitrogênio e proteína, aumentando sua atratividade em relação aos macroinvertebrados.

O processo de decomposição se dá principalmente pela ação de bactérias e fungos, os quais convertem fibras e paredes celulares em matéria saprofítica e gás carbônico. $O$ aumento da concentração de nitrogênio ocorre a partir do trabalho da microflora epifítica (Suren \& Lake, 1989).

A família Chironomidae tem sido reportada como um dos principais grupos de macroinvertebrados colonizadores de macrófitas no processo de decomposição (Trivinho-Strixino \& Strixino, 1993; Grubbs et al., 1995; Gonçalves-Jr., 1999). Estudos anteriores realizados por Nessimian (1995 a, b) e Nessimian \& Sanseverino (1995) registraram a presença de ao menos 15 gêneros desta família, no Brejo-canal de Itaipuaçu, um brejo de dunas em Maricá no litoral do Estado do Rio de Janeiro e mostraram um maior índice de ocupação por este grupo após a senescência da macrófita dominante, Eleocharis sellowiana (Cyperaceae). Nessimian \& De Lima (1997) em um experimento de colonização de três macrófitas no mesmo corpo d'água verificaram que larvas de Chironomidae constituíram o principal grupo com $58 \%$ do total de indivíduos.

Este estudo visa observar a colonização de Eleocharis sellowiana por larvas de Chironomidae durante o processo de decomposição.

\section{Área de Estudos}

O presente estudo foi realizado no Brejo-canal de Itaipuaçu, em Maricá, $43^{\circ} 54^{\prime} \mathrm{W} ; 22^{\circ} 57^{\prime} \mathrm{S}$, Estado do Rio de Janeiro. O clima da região é 
do tipo Aw de Köppen, com verão chuvoso e quente e inverno seco. A precipitação média anual situa-se entre $1000 \mathrm{~mm}$ e $1350 \mathrm{~mm}$. (Franco et al., 1984).

O brejo situa-se entre dois cordões arenosos da restinga e possui cerca de 1500 m de extensão e uma largura máxima de 200 m, sendo sua formação resultado do fechamento de um canal que ligava a lagoa ao mar. Possui pequena profundidade, águas pretas e ácidas e substrato compacto de material orgânico sobre solo arenoso. Possui caráter semi-permanente, sendo a variação do nível da água relacionada à do lençol freático, às chuvas e à evaporação. O nível máximo é alcançado no inverno e o mínimo no verão (Carmo \& Lacerda, 1984; Nessimian, 1995a).

A vegetação dominante é constituída por Eleocharis sellowiana Kunth. (Cyperaceae). Outras macrófitas presentes são: Rhynchosphora corymbosa (L.) Britton (Cyperaceae), Sagittaria lancifolia L. (Alismataceae), Nymphoides indica (L.) O.Kuntze (Menyanthaceae), Utricularia gibba L. (Lentibulariaceae) e Ludwigia longifolia (D.C.) Hara (Onagraceae). Em determinados períodos é marcante a presença de grandes quantidades de algas clorófitas do gênero Siyrogyra.

\section{Material e Métodos}

Os dados gerais deste estudo são provenientes de um experimento de colonização de três espécies de macrófita por macroinvertebrados realizado por Nessimian e De Lima (1997), no qual não foram estudados os Chironomidae.

Para o estudo de colonização de litter de Eleocharis sellowiana foram pesados lotes de $50 \mathrm{~g}$ de folhas frescas, sendo estas lavadas e colocadas em sacos de filó com malha de 1,5 mm de abertura. Foram preparadas 25 amostras e dispostas no corpo d'água em 5 transectos paralelos. 0 experimento teve duração de 70 dias (abril-junho de 1996). As retiradas foram efetuadas em 7, 14, 28, 42 e 70 dias após o início do experimento, sendo o material coletado sempre no mesmo horário. As amostras foram fixadas em formaldeído a $4 \%$ e no mesmo dia, lavadas, sendo o material retido em uma peneira com malha de $0,21 \mathrm{~mm}$ de abertura e posteriormente conservado em álcool etílico a $80 \%$.

Os macroinvertebrados presentes foram triados e os Chironomidae separados sob estereomicroscópio com aumento de até 160 vezes. As 
folhas foram separadas, secas e pesadas em balança com precisão de 1 mg. Foram realizadas observações quanto ao estado de decomposição. As larvas de Chironomidae foram montadas em lâminas permanentes e identificadas sob microscópio óptico com aumento de até 400 vezes. Para a identificação, até o nível de gênero, foram consultadas as obras de Cranston et al. (1983), Epler (1995) e Trivinho-Strixino \& Strixino (1995). Observações de conteúdo digestivo foram realizadas em material fixado sob microscópio.

Dados fisico-químicos da água (condutividade elétrica, $\mathrm{pH}$, oxigênio dissolvido e temperatura) foram tomados mensalmente (abril, maio e junho), assim como a variação da coluna d'água. $O$ teor de oxigênio dissolvido foi medido pelo método de Winkler (Brower \& Zar, 1977).

Associações possíveis entre os diversos táxons encontrados foram determinadas através do coeficiente de correlação de Pearson e posteriormente através de regressão múltipla e aceitas para $\mathrm{p}<0,05$ (Sokal \& Rohlf, 1981).

\section{Resultados}

De acordo com os estudos de Carmo \& Lacerda (1984) e Nessimian (1995b) o período de elevação do nível de água, no qual foi realizado o experimento, caracteriza-se por uma queda nos valores de condutividade elétrica, temperatura e pH e uma elevação nos teores de oxigênio dissolvido, o que não foi observado para os dois últimos fatores (Tab. I).

Após 70 dias de experimento, foram amostrados 7688 indivíduos de macroinvertebrados dos quais Insecta representaram $51 \%$, sendo que

\section{Tabela I}

Variáveis ambientais medidas no Brejo-canal de Itaipuaçu, Maricá, nos meses de abril, maio e junho de 1996.

\begin{tabular}{lcccccc}
\hline & $\begin{array}{c}\text { OD } \\
\left(\mathrm{mg}^{-1} \mathrm{I}^{-1}\right)\end{array}$ & $\begin{array}{c}\text { CE } \\
\left(\mu \mathrm{S} . \mathrm{cm}^{-1}\right)\end{array}$ & $\mathbf{p H}$ & $\begin{array}{c}\text { TC } \\
\left({ }^{\circ} \mathrm{C}\right)\end{array}$ & $\begin{array}{c}\text { TH } \\
\left({ }^{\circ} \mathrm{C}\right)\end{array}$ & $\begin{array}{c}\text { PF } \\
(\mathrm{cm})\end{array}$ \\
\hline Abril & 5,42 & 158,0 & 4,7 & 27,0 & 28,5 & 38,1 \\
Maio & 4,14 & 142,5 & 4,0 & 26,5 & 27,5 & 55,8 \\
Junho & 4,49 & 110,5 & 4,9 & 26,7 & 24,5 & 61,3 \\
\hline
\end{tabular}

CE: condutividade elétrica; OD: oxigênio dissolvido; $\mathrm{pH}$ : potencial hidrogeniônico; TC: temperatura do ar; TH: temperatura da água; PF: altura da coluna d'água. 
destes, os Chironomidae (Diptera) constituíram o principal táxon com $58 \%$, totalizando 2347 indivíduos.

Dentre os Chironomidae, Chironomini (Chironominae) constituiu o grupo mais abundante com $63,1 \%$, seguido de Tanypodinae com $34,3 \%$. Tanytarsini (Chironominae) apresentou participação reduzida (2,6\%). Os gêneros encontrados foram Chironomus, Polypedilum, Beardius (Chironomini), Tanytarsus (Tanitarsini), Clinotanypus, Ablabesmyia, Labrundinia e Larsia(?) (Tanypodinae). Chironomus foi o grupo mais importante com mais de $50 \%$ do total, seguido de Larsia (?) com aproximadamente $25 \%$.

Os Chironomini, especialmente Beardius e Chironomus apresentaram abundância crescente até 42 dias de exposição, havendo uma pequena queda ao final do experimento. O mesmo crescimento ocorreu com os Tanypodinae, havendo uma estabilização após 42 dias. Eleocharis sellowiana não apresentou perda visível em sua estrutura durante o período de exposição. Suas folhas tornaram-se escuras e perderam a rigidez lentamente, sendo o processo mais acentuado a partir de 28 dias. No fim do experimento houve perda de cerca de $40 \%$ do peso inicial. Com relação à densidade populacional, houve aumento significativo da quantidade de larvas presentes por peso remanescente da macrófita. Quanto à participação relativa de cada gênero, houve um aumento de Chironomus em direção ao final do período de estudo, ocorrendo o oposto com Larsia (?), Labrundinia e Tanytarsus. Beardius, Polypedilum e Clinotanypus não apresentaram variação (Figs. 1-4)

A maior parte dos táxons apresentou algas desmidiáceas como principal item alimentar. Ablabesmyia e Labrundinia apresentaram também, restos animais (Crustacea, Acari, Chironomidae) em seus conteúdos. Polypedilum, Beardius e Chironomus mostraram grande quantidade de detritos orgânicos.

Houve uma associação positiva entre Chironomus, Beardius, Polypedilum e Larsia (?). Esses grupos relacionaram-se negativamente com os dois principais táxons predadores dentre os Chironomidae (Ablabesmyia e Labrundinia). Com relação a outros grupos de macroinvertebrados, houve correlação positiva de táxons de hábitos coletores, incluindo Chironomus, Beardius, Polypedilum e Larsia (?) (também predadores) com Ostracoda, Copepoda, Oxyethira hyalina (Hydroptilidae) e Berosus (Hydrophilidae). Por outro lado, houve correlação negativa entre estes grupos de Chironomidae e grupos predadores como Odonata, Naucoridae e Belostomatidae, embora não significativas para $\mathrm{p}<0,05$ (Tab. II). 


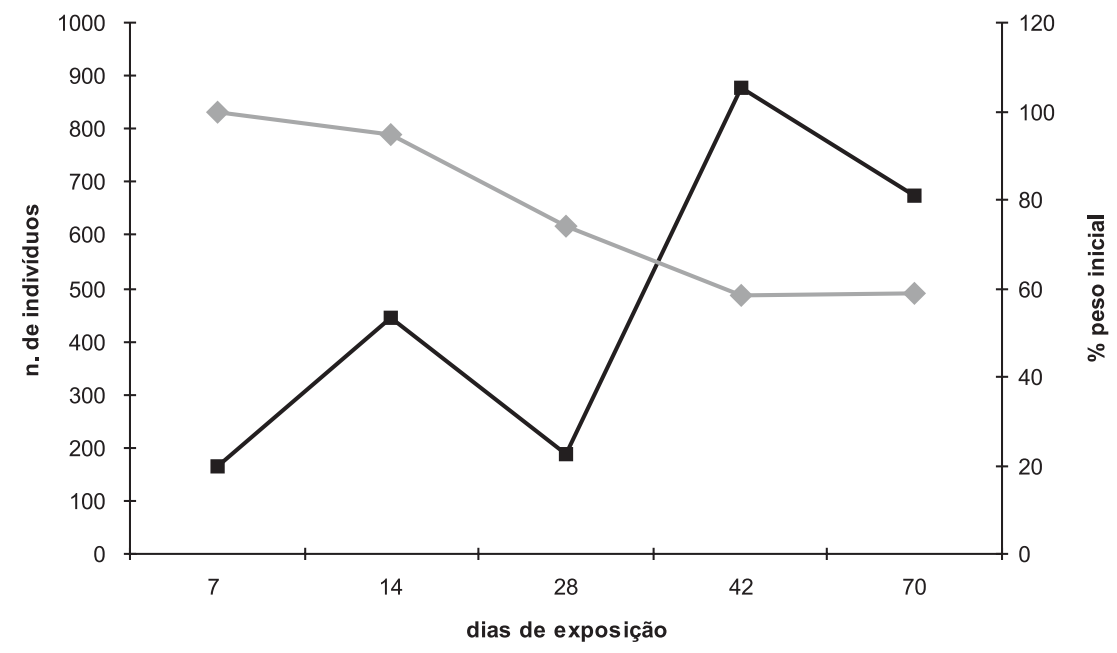

$\rightarrow-$ número de larvas $\multimap$ E.sellowiana

Figura 1: Variação do número de larvas de Chironomidae coligidas e perda de peso de Eleocharis sellowiana (\%) durante o período de exposição de 70 dias.

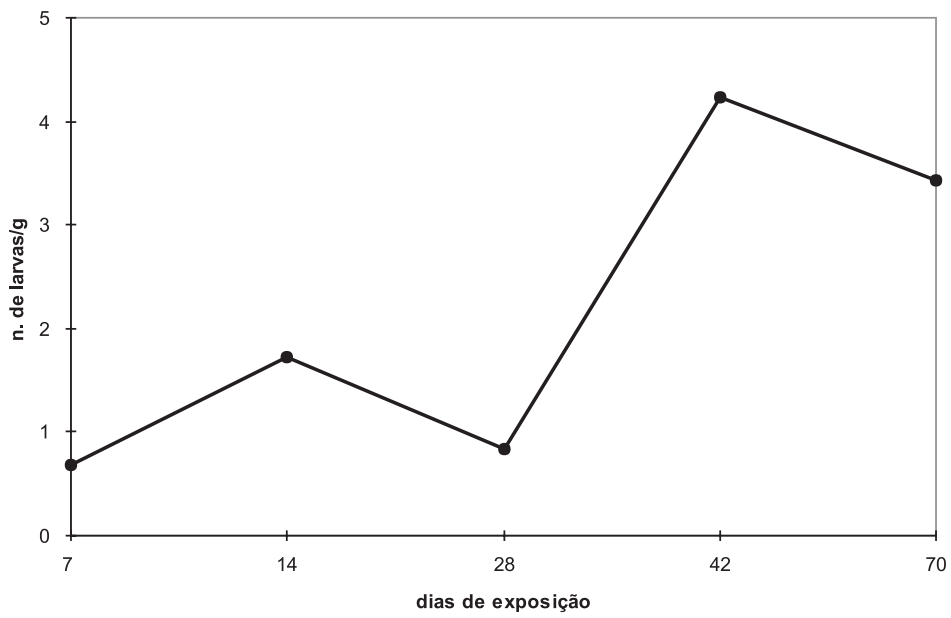

$\multimap$ número de larvas por grama de $\mathrm{E}$. sellowiana

Figura 2: Número de larvas de Chironomidae por grama de peso de $E$. sellowiana durante o período de 70 dias de exposição. 


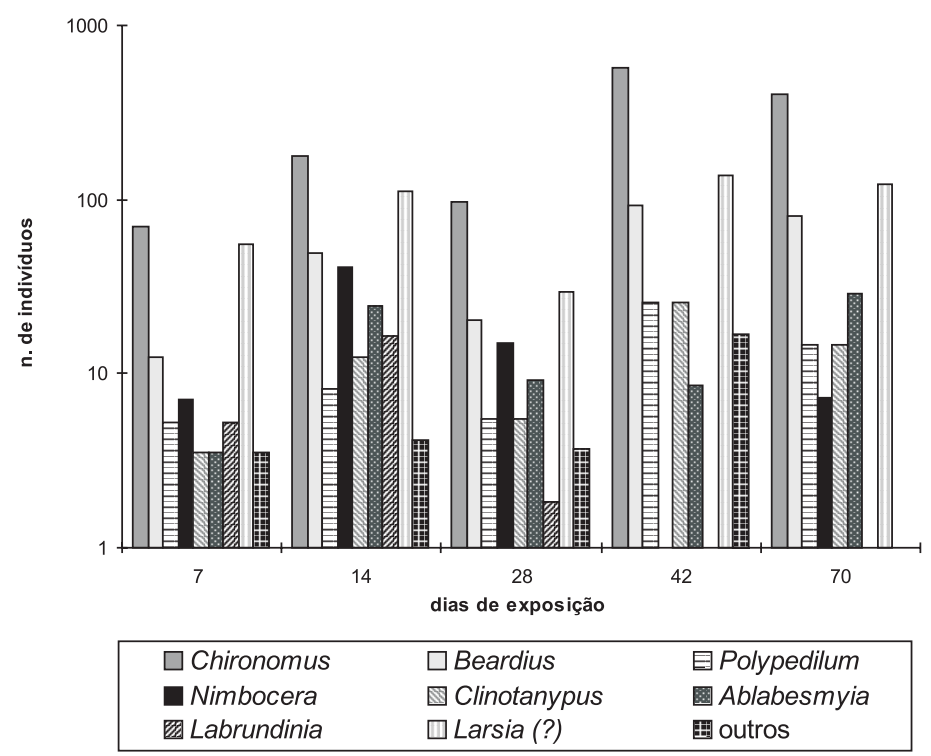

Figura 3: Número de larvas de gêneros presentes de Chironomidae coligidas durante no período de 70 dias de exposição de litter de Eleocharis sellowiana.

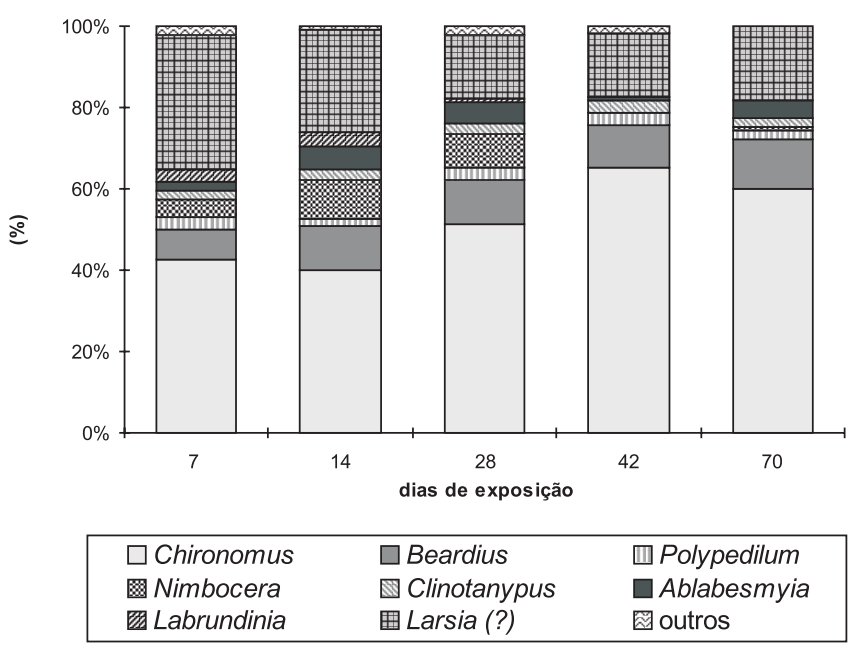

Figura 4: Participação relativa dos gêneros presentes de Chironomidae durante no período de 70 dias de exposição de litter de Eleocharis sellowiana. 


\section{Tabela II}

Correlações acima de 0,40 entre Chironomidae e outros grupos de macroinvertebrados durante o experimento de colonização do litter de Eleocharis sellowiana (Valores significativos para $p<0,05$ e $n=5$ estão em negrito).

\begin{tabular}{|c|c|c|c|c|c|c|c|c|c|}
\hline & Ch & $\mathrm{Bd}$ & Po & $\mathrm{Ta}$ & $\mathbf{C t}$ & $\mathbf{A b}$ & Lb & La & Tp \\
\hline Chironomus & 1,00 & 0,97 & 0,97 & $-0,47$ & 0,95 & & $-0,46$ & 0,85 & 0,61 \\
\hline Beardius & 0,97 & 1,00 & 0,90 & & 0,93 & 0,47 & & 0,92 & 0,47 \\
\hline Polypedilum & 0,97 & 0,90 & 1,00 & $-0,50$ & 0,97 & & $-0,46$ & 0,77 & 0,78 \\
\hline $\begin{array}{l}\text { Tanytarsus } \\
\text { rhabdomanthis }\end{array}$ & $-0,47$ & & $-0,50$ & 1,00 & & 0,46 & 0,93 & & \\
\hline Clinotanypus & 0,95 & 0,93 & 0,97 & & 1,00 & & & 0,85 & 0,76 \\
\hline Ablabesmyia & & 0,47 & & 0,46 & & 1,00 & & 0,56 & $-0,47$ \\
\hline Labrundinia & $-0,46$ & & $-0,46$ & 0,93 & & & 1,00 & & \\
\hline c.f. Larsia & 0,85 & 0,92 & 0,77 & & 0,85 & 0,56 & & 1,00 & \\
\hline Tanypodinae & 0,61 & 0,47 & 0,78 & & 0,76 & $-0,47$ & & & 1,00 \\
\hline Naididae & $-0,44$ & $-0,40$ & & 0,42 & & & 0,71 & & \\
\hline Acari & 0,81 & 0,68 & 0,80 & $-0,83$ & 0,66 & & $-0,89$ & 0,40 & 0,49 \\
\hline Ostracoda & 0,99 & 0,89 & 0,98 & $-0,52$ & 0,94 & & $-0,56$ & 0,68 & 0,73 \\
\hline Cladocera & $-0,49$ & $-0,59$ & $-0,43$ & & $-0,53$ & $-0,54$ & & $-0,40$ & \\
\hline Copepoda & $-0,46$ & $-0,55$ & $-0,42$ & & $-0,51$ & $-0,48$ & & & \\
\hline Caenis cunniana & & & 0,52 & & 0,54 & & & & 0,71 \\
\hline Odonata & $-0,44$ & & $-0,43$ & 0,51 & & & 0,77 & & \\
\hline Naucoridae & $-0,40$ & & $-0,46$ & 0,91 & & 0,53 & 0,70 & & $-0,44$ \\
\hline Belostomatidae & $-0,57$ & $-0,65$ & $-0,62$ & & $-0,77$ & & $-0,41$ & $-0,77$ & $-0,60$ \\
\hline Noteridae & & & & & & 0,54 & $-0,49$ & & $-0,58$ \\
\hline Dytiscidae & 0,50 & 0,56 & & & & 0,66 & $-0,51$ & 0,42 & \\
\hline Hydrophilidae & 0,79 & 0,67 & 0,91 & $-0,49$ & 0,85 & & & 0,55 & 0,96 \\
\hline Pyralidae & & & 0,49 & & 0,49 & 0,64 & & & 0,92 \\
\hline Oxyethira hyalina & 0,98 & 0,94 & 0,97 & $-0,49$ & 0,95 & & $-0,53$ & 0,77 & 0,65 \\
\hline Ceratopogonidae & & & & & & 0,94 & & 0,52 & $-0,59$ \\
\hline Tabanidae & $-0,57$ & $-0,51$ & $-0,47$ & 0,69 & & & 0,56 & $-0,51$ & \\
\hline
\end{tabular}

Ch: Chironomus; Bd: Beardius; Po: Polypedilum; Ta: T. rhabdomanthis; Ct: Clinotanypus; Ab: Ablabesmyia; Lb: Labrundinia; La: c.f. Larsia; Tp:Tanypodinae. 


\section{Discussão}

A perda de peso observada em E. sellowiana foi semelhante à obtida por Carmo (1984) em estudo sobre a decomposição desta espécie no mesmo corpo d'água, sendo lenta a decomposição dos componentes estruturais.

Com relação à colonização os padrões observados, em termos de sucessão, foram semelhantes aos dados obtidos por Bruquetas de Zozaya \& Neiff (1991) em um brejo na Argentina, para diversos grupos presentes. Nessimian e de Lima (1997) apontam a pequena participação de Chironomidae, no início, comparada à de microcrustáceos e ácaros. Porém, especialmente cladóceros e copépodos diminuíram sua participação logo após a primeira semana de exposição, ocorrendo o oposto com Chironomidae. Estudos realizados no mesmo corpo d'água (Nessimian, 1995 b), mostram que o período de elevação do nível d'água caracteriza-se pela ocupação do brejo pelos primeiros colonizadores. Cladocera, Ostracoda e Acari são muito abundantes neste período. O período final do outono e início do inverno é marcado pelo aparecimento de Parapoynx restingalis (Pyralidae), Caenis. cunniana (Caenidae) e Tanytarsus. rhabdomantis e uma queda na freqüência de Cladocera e Copepoda. O mesmo padrão foi observado na ocupação de E. sellowiana. Segundo Oliver (1971) Chironomidae são capazes de eficiente dispersão, através de oviposição e/ou da capacidade de nado das larvas de primeiros ínstares (pelágicas), colonizando assim novos habitats com facilidade. Contudo, não foram encontrados alguns grupos normalmente presentes como colonizadores de Eleocharis sellowiana. Dos 15 gêneros registrados (Nessimian, 1995 a, b; Nessimian \& Sanseverino, 1995) somente cinco ocorreram. Alguns abundantes como Ablabesmyia tiveram participação pequena e somente com larvas menores. Outro grupo comum no período limnológico em que se realizou o estudo, Zavreliella, não ocorreu. Isso confirma, como assinalado por Nessimian \& Sanseverino (1995), que tais espécies são preferencialmente habitantes do substrato e o fato do material exposto ter permanecido na superfície e sem ligação com o fundo, impediu uma colonização efetiva. Clinotanypus, embora ocupe efetivamente o fundo, possui boa capacidade de nado, ocorrendo nas amostras com maior freqüência. Trivinho-Strixino e Strixino (1993) em um estudo acerca da fauna associada a Pontederia lanceolata no Estado de São Paulo demonstraram que a região das raízes suporta a fauna mais abundante que em outras partes da planta. Os resultados também confirmam observações anteriores 
de períodos de ocorrência para alguns grupos (Nessimian, 1995 b). Por exemplo, Djalmabatista, cujas larvas tem boa capaciadade de nado, tem sua principal ocorrência, no brejo, no período de águas baixas, o que explicaria a ausência deste grupo no experimento.

Os resultados confirmam as observações anteriores de que a importância das macrófitas vivas como fonte de alimento para macroinvertebrados é muito pequena devido à sua pouca digestibilidade (Smock \& Stoneburner, 1980; Trivinho-Strixino \& Strixino, 1993). De acordo com Suren \& Lake (1989), bactérias e fungos e posteriormente algas epifíticas, durante o processo de decomposição, promovem um aumento nas concentrações de nitrogênio e proteínas resultando em um aumento da atratividade em relação aos macroinvertebrados. Tal fato foi observado no Brejo-canal de Itaipuaçu por Nessimian (1995b) e Da Silva (1994), onde a maior concentração de macroinvertebrados ocorreu após a morte das folhas de E. sellowiana, macrófita dominante e de ciclo anual. Dos Chironomidae presentes, observações feitas acerca da alimentação demonstram que algas, principalmente desmidiáceas foram os ítens alimentares mais encontrados, incluíndo a maioria dos Tanypodinae (especialmente Larsia e Clinotanypus). De acordo com Trivinho-Strixino \& Strixino (1993) ocorre uma maior concentração de retalhadores nas partes mais velhas e em decomposição da planta e uma presença maciça de coletores entre as raízes, por estarem em contato com o substrato e favorecer o acúmulo de detritos. No presente estudo Chironomus e Beardius, além de algas, apresentaram grandes quantidades de detrito, o que pode estar relacionado ao processo de decomposição.

Os itens alimentares encontrados estão de acordo com os observados por Nessimian et al. (1999). Grupos de mesmos hábitos alimentares apresentaram correlações positivas caracterizando uma sobreposição de nicho alimentar. Segundo Tokeshi (1986), este fato está relacionado à limitação de recursos em termos de variedade. O mesmo autor mostra que a competição por recurso alimentar não é fator importante na organização temporal da comunidade de Chironomidae epifíticos. Relações negativas foram observadas quanto a grupos predadores para vários Chironomidae, 0 que confirma a distribuição, sobre várias espécies, do impacto de predação assinalada por Nessimian (1997). Contudo, este autor, em seu estudo, com amostragens mensais não detectou tais relações. Estes resultados, aparentemente conflitantes, mostram que em curto espaço de tempo há uma recuperação das populações das espécies usadas como alimento. 


\section{Agradecimentos}

Os autores agradecem ao Prof. Gabriel Mejdalane (Museu Nacional, UFRJ) pela revisão do artigo.

\section{Referências Bibliográficas}

Brower, J.E. \& Zar, J.H., 1977. Field and Laboratory Methods for General Ecology. Wm. C. Brown pub., Dubuque, 2nd ed. 226 p.

Bruquetas de Zozaya, I.Y. \& Neiff, J.J., 1991. Decomposition and colonization by invertebrates of Typha latifolia L. litter in Chaco cattail swamp (Argentina). Aquat.Bot. 40:185-193.

Carmo, M.A.M., 1984. O papel de Eleocharis subarticulata (Nees) Boeckler (Cyperaceae) na ciclagem de um brejo da Restinga de Maricá, RJ. Dissertação de mestrado, Universidade Federal Fluminense, Niterói, $100 \mathrm{p}$.

Carmo, M.A.M. \& Lacerda, L.D., 1984. Limnologia de um brejo de dunas em Maricá, RJ. p. 453-458. In: Restingas: origens, estruturas, processos. Lacerda, L.D.; Araújo, D.S.D.; Cerqueira, R. \& Turcq, B. orgs. - Niterói, CEUFF, Universidade Federal Fluminense.

Cranston, P.S., Oliver, D.R. \& Sæther, O.A., 1983. The larvae of Orthocladiinae (Diptera: Chironomidae) of the Holartic region - keys and diagnoses. Entomol. Scand. Suppl. 9:149-291.

Da Silva, E.R., 1994. Aspectos da biologia de Callibaetis guttatus Navás, 1915 (Insecta: Ephemeroptera: Baetidae) em alagados temporários da restinga de Barra de Maricá, Estado do Rio de Janeiro, com considerações taxonômicas. Dissertação de mestrado. Museu Nacional, UFRJ, Rio de Janeiro. 109 p.

Epler, J.H., 1995. Identification Manual for the Larvae Chironomidae (Diptera) of Florida. Departament of environmental Protection. Division of water facilities Tallahasse. $308 \mathrm{p}$. 
Franco, A.C.; Valeriano, D.M.; Santos, F.M.; Henriques, R.P.B.; Hay, J.D. \& Medeiros, R.A., 1984. Os microclimas das zonas de vegetação da praia da restinga de Barra de Maricá, Rio de Janeiro. p. 327-342. In: Restingas: origens, estruturas, processos. Lacerda, L.D.; Araújo, D.S.D.; Cerqueira, R. \& Turcq, B. orgs. - Niterói, CEUFF, Universidade Federal Fluminense.

Nessimian, J.L., 1995a. Composição da fauna de invertebrados bentônicos em um brejo entre dunas no litoral do Estado do Rio de Janeiro. Acta Limnol. Bras. 7:41-59.

Nessimian, J.L. 1995b. Abundância e biomassa de macroinvertebrados bentônicos em um brejo de dunas no litoral do Estado do Rio de Janeiro. Rev. Bras. Biol. 55:661-683.

Nessimian, J.L., 1997. Categorização funcional trófica da fauna de macrinvertebrados em um brejo no litoral do Estado do Rio de Janeiro. Rev. Bras. Biol. 57:135-145.

Nessimian, J.L. \& De Lima, I.H.A.G., 1997. Colonização de três espécies de macrófitas por macrinvertebrados aquáticos em um brejo no litoral do Estado do Rio de Janeiro. Acta. Limnol. Bras. 9:149-163.

Nessimian, J.L.; Sanseverino, A.M. \& Oliveira, A.L.H., 1999. Relações tróficas de larvas de Chironomidae e sua importância na rede alimentar em um brejo no litoral do Estado do Rio de Janeiro. Rev. Bras. Entomol 43:47-53.

Oliver, D.R., 1971. Life history of the Chironomidae. Ann. Rev. Entomol.12:211-230.

Smock, L.A. \& Stoneburner, D.L., 1980. The response of macroinvertebrate to aquatic macrophyte decomposition. Oikos 35:397-403.

Suren, A.M. \& Lake, P.L., 1989. Edibility of fresh and decomposing macrophytes to three species of freshwater invertebrate herbivores. Hydrobiologia 178:165-178. 
Tokeshi, M., 1986. Resource utilization, overlap and temporal community dynamics: A null model analysis of na epiphytic chironomid community. J. Anim. Ecol. 55:491-506.

Trivinho-Strixino, S. \& Strixino, G., 1993. Estrutura da comunidade de insetos aquáticos associados à Pontederia lanceolata Nuttal. Rev. Bras. Biol. 53:103-111.

Trivinho-Strixino, S. \& Strixino, G., 1995. Larvas de Chironomidae (Diptera) do Estado de São Paulo - Guia de identificação e diagnose dos gêneros. PPG-ERN/UFSCAR, São Carlos. 229 p.

Voigts, D.K., 1976. Aquatic invertebrate abundance in relation to changing marsh vegetation. Am. Midl. Nat. 95:313-322. 